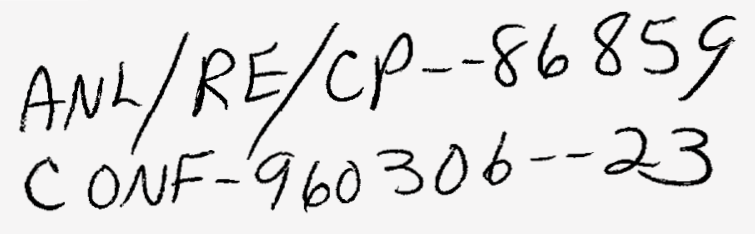

Paper to be presented at the 4th International Conference on Nuclear Engineering, New Orleans, USA, March 10-14, 1996

Sponsored by: ASME and JSME

\title{
PRODIAG - A HYBRID ARTIFICIAL INTELLIGENCE BASED REACTOR DIAGNOSTIC SYSTEM FOR PROCESS FAULTS
}

\author{
J. Reifman, T. Y. C. Wel and J. E. Vitela \\ Argonne National Laboratory \\ Argonne, llinois \\ C. A. Applequist \\ Commonwealth Research Corporation \\ Chicago, Illinois \\ T. M. Chasensky \\ Commonwealth Edison Company \\ Braidwood, Illinois
}

\section{DISCLAIMER}

\begin{abstract}
This report was prepared as an account of work sponsored by an agency of the United States Government. Neither the United States Government nor any agency thereof, nor any of their employees, makes any warranty, express or implied, or assumes any legal liability or responsibility for the accuracy, completeness, or usefulness of any information, apparatus, product, or process disclosed, or represents that its use would not infringe privately owned rights. Reference herein to any specific commercial product, process, or service by trade name, trademark, manufacturer, or otherwise does not necessarily constitute or imply its endorsement, recommendation, or favoring by the United States Government or any agency thereof. The views and opinions of authors expressed herein do not necessarily state or reflect those of the United States Government or any agency thereof.
\end{abstract}

*Work supported by the U.S. Department of Energy, Energy Research-Laboratory Technology Transfer Program, under contract W-31-109-ENG-38. 


\title{
PRODIAG - A HYBRID ARTIFICIAL INTELLIGENCE BASED REACTOR DIAGNOSTIC SYSTEM FOR PROCESS FAULTS
}

\author{
J. Reifman, T. Y. C. Wei and J. E. Vitela \\ Argonne National Laboratory \\ Argonne, Illinois \\ C. A. Applequist \\ Commonwealth Research Corporation \\ Chicago, Illinois \\ T. M. Chasensky \\ Commonwealth Edison Company \\ Braidwood, llinois
}

\section{INTRODUCTION}

Commonwealth Research Corporation (CRC) and Argonne National Laboratory (ANL) are collaborating on a DOEsponsored Cooperative Research and Development Agreement (CRADA) project to perform feasibility studies on a novel approach to Artificial Intelligence (AI) based diagnostics for component faults in nuclear power plants. Investigations are being performed in the construction of a first-principles physicsbased plant level process diagnostic expert system (ES) and the identification of component-level fault patterns through operating component characteristics using artificial neural networks (ANNs). The purpose of the proof-of-concept project is to develop a computer-based system using this AI approach to assist process plant operators during off-normal plant conditions. The proposed computer-based system will use thermal hydraulic (T-H) signals complemented by other non-T-H signals available in the data stream to provide the process operator with the component which most likely caused the observed process disturbance. To demonstrate the scale-up feasibility of the proposed diagnostic system it is being developed for use with the Chemical Volume Control System (CVCS) of a nuclear power plant. A full-scope operator training simulator representing the Commonwealth Edison Braidwood nuclear power plant is being used both as the source of development data and as the means to evaluate the advantages of the proposed diagnostic system.
This is an ongoing multi-year project and this paper presents the results to date of the CRADA phase.

The novel AI diagnostics approach has been implemented in the PRODIAG code. The function of the PRODIAG code is to diagnose on-line the root cause of a T-H system transient with trace back to the identification of the malfunctioning component using the $\mathbf{T}-\mathrm{H}$ instrumentation signals exclusively. The code methodology is based on the AI techniques of automated reasoning through an ES and pattern recognition through ANNs. The research and development objective is to develop a generic code methodology which would be plant- and T-H-systemindependent. The only plant or T-H system specific code requirements would be implemented through input only, and at that only through a Piping and Instrumentation Diagram (PID) database (Reifman et al., 1994 and 1995a). PRODIAG is, therefore, generic and portable from $\mathrm{T}-\mathrm{H}$ system to $\mathrm{T}-\mathrm{H}$ system and from plant to plant without requiring any code modifications except for the PID database. This gives PRODLAG the generic feature which numerical simulation plant codes such as TRAC or RELAP5 have. As the code is applied to different plants and different T-H systems, only the connectivity information and the operating conditions are changed, and the changes are made entirely through input. Verification and validation of PRODIAG is, therefore, plant and T-H system-independent and needs to be performed only "once". With each different application, 
verification needs only to be performed for the input deck which is the specific T-H system model (Reifman et al., 1994 and 1995a).

To achieve the driving technical objective of a generic code, several novel theoretical concepts in intelligent database knowledge structuring had to be introduced and developed in detail. The PRODIAG Theory Manual, Volume 1, describes those concepts and those details (Reifman et al., 1995). It forms the basis for the practical implementation of the diagnostic algorithm as described in the PRODIAG Code Manual, Volume 2 (Reifman et al., 1995). In summary, in contrast to a traditional event-oriented knowledge base (Bernard and Washio, 1989; Kirk and Murray, 1988); the knowledge structure used by PRODIAG is based on T-H first-principles (Reifman et al., 1991a; Reifman et al., 1991b). System functions (such as heat or mass transfer) are used in conjunction with equipment characteristics (such as pressure-versus-flow curves that define the operating ranges of pumps and valves). Together, these provide a two-level approach to diagnosing process system faults. At the first level, an expert system uses $\mathrm{T}-\mathrm{H}$ functions to determine physical occurrences (i.e., water added or lost, heat added or lost). At the second level, artificial neural networks pinpoint the source of the transient by classifying the functional misbehavior of the system through specific component characteristics.

The current status of PRODIAG is that the conceptual approach with the overall system design has been defined. Implementation has been carried out largely for those knowledged based structure concepts which were necessary for the Braidwood CVCS test problem. The focus has been mostly on the ES part. Due to the limited availability of equipment characteristics data, the implementation of the second level of the diagnostic strategy using ANNs has been deferred to future work. The ANN implementation which has been performed has been mainly for the purpose of supplementing the ES part of the diagnostic strategy. Those knowledge based structuring concepts which have been developed for the current laboratory-scale version of PRODIAG have been implemented for the following range of applicability:

- single-phase liquid plus noncondensible gas T-H systems;

- non-neutronic heat sources;

- coolants with bulk moduli and thermal expansion coefficients similar to water;

- single-fault initiated transient scenarios;

- transient severity should be sufficient for instrumentation in single-phase liquid components to respond;

- use of instrumentation signal data which has been filtered for noise;

- T-H instrumentation; flow $w$, pressure $p$, temperature $T$, and level $\mathbf{\ell}$; and

- diagnostic window closure upon initiation of control action.

The next section describes the overall diagnostic strategy which forms the framework for the two-level hierarchical structuring of the PRODIAG knowledge database.

\section{DIAGNOSTIC STRATEGY THEORY}

Decades ago when numerical computer simulation of thermalhydraulic systems was being introduced, a different simulation code was written or "hard-wired" for each thermal-hydraulic system. It was then realized that the universal conservation equations permitted the usage of basic elements, volumes, junctions/pipes and heat slabs which then allowed the development of a "few" simulation codes which could model a wide variety of thermal-hydraulic systems. The field of expert systems for process diagnostics has reached a similar point. Canonical structuring of this T-H knowledge base into an ES process diagnostics language with a number of key abstractions and general concepts transformed into language constructs (mass source, energy sink, ..., momentum imbalance), allows this generic thermal-hydraulic system-independent generalization. The canonical structuring has to be grounded on the universal mathematical description. If not, heuristics would inevitably creep in and render the concept of thermal-hydraulic system independence meaningless. The mathematical description has to be transformed into the AI system construct of rules, representations, and supervisory flow logic (Reifman and Wei, 1995a). The PRODIAG approach is a first-principles approach. The mathematical description consists of the three (mass, momentum, and energy) conservation equations, transport properties, correlations for viscosities and conductivity and, usually, the pressure equation of state. We divide the transformation into parts by using the following taxonomy for $\mathrm{T}$ $H$ systems which assumes no chemical reactions: (1) fluid material, (2) phase of matter, (3) geometrical configuration, and (4) operation condition.

At this stage of the research, we choose to focus on subcooled liquid water. Future research will reexamine the effect of fluid material and phase selection on the generalization of the expert system. Currently, it appears that the bulk modulus and the thermal expansion coefficient are the only fluid material properties which affect the generalization for subcooled liquids. The bulk modulus determines incompressibility which is extensively used in the transformation of the mathematical description. A low thermal expansion coefficient allows the use of the decoupling of the energy equations from the mass and momentum equations in the transformation. This still leaves the effect of geometrical configuration and operating condition. To permit the generalization, the taxonomy is broken down further in the following section.

Table 1 shows the structure of the analytic decomposition strategy. An overall strategy is necessary to allow the generic portability from T-H system to T-H system without major code modifications. Every component in a $\mathrm{T}-\mathrm{H}$ system has a dominant $\mathrm{T}-\mathrm{H}$ function and $\mathrm{T}-\mathrm{H}$ characteristics. Table 1 summarizes the mathematical formulas by classes used to describe this T-H physics. These classes can be used to classify the $\mathrm{T}-\mathrm{H}$ components by key general features. However, they also indicate that specific components have $\mathrm{T}-\mathrm{H}$ signatures which are component specific. The first two columns of Table 1 show the connection between the component classification/diagnostic 
TABLE 1. ANALYTIC DECOMPOSITION STRATEGY TREE STRUCTURE

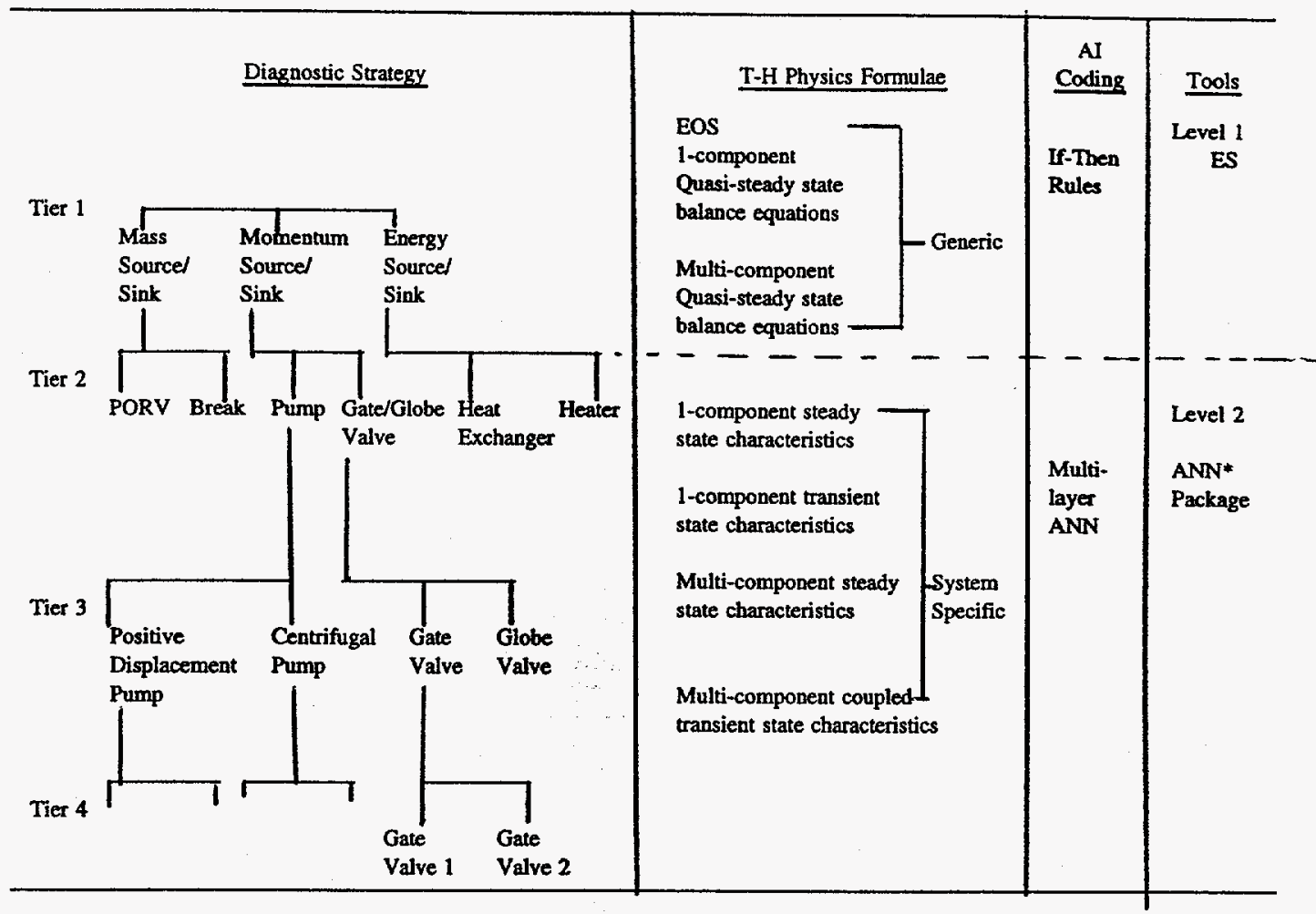

*Due to limited availability of component characteristics data, implementation deferred to future work.

strategy and the T-H physics formulae. The quasi steady conservation equations/equation of state (EOS) basically describe the first order temporal trend behavior of the $\mathrm{T}-\mathrm{H}$ function of the component or set of components which is a generic feature. This physics suggests that the diagnostic strategy should be to first classify the components by mass source/sink $\left(Q_{\text {mass }}\right)$ function class, momentum source/sink $\left(Q_{\text {mom }}\right)$ function class or energy source/sink ( $Q_{\text {eng }}$ ) function class. The component "steady" state operating characteristics describes the low frequency temporal behavior of the component which can have generic or component specific features. The next step in the diagnostic strategy is then to classify between generic components such as pumps or valves within a $Q$ function class. Finally the component transient operating characteristics describes the high frequency temporal behavior of the component which is usually a component specific feature. Final classification should then be between specific components such as valve 1 or valve 2 within a generic component class.

The overall diagnostic strategy is summarized in three steps: (1) Use a prescribed formula with a set of the T-H signal data to detect the presence or absence of a key T-H feature; then, (2) check a predetermined classification list of components classified by the key $\mathrm{T}-\mathrm{H}$ feature to obtain a candidate list of potential malfunctioning components using the previously detected present or absent key T-H features; then, (3) check the system connectivity information contained in the PID to see what match and reduction can be made against the previous candidate list of potential malfunctioning components. If a single unique match can be made, then the malfunctioning component has been identified. Otherwise, repeat the three step process with other formulas and other key T-H features. The following three sets of T-H formulas are used with the set of T-H signal data to detect the presence or absence of key T-H features. There are, in order of diagnostic sequence: (1) The three conservation equations of mass, momentum, and energy to detect changes in the features of the three key T-H functions; mass, momentum, and energy transfer; (2) the normal operation quasi-static component $\mathrm{T}-\mathrm{H}$ characteristics to detect generic and specific component characteristics changes; and (3) the normal operation transient component $\mathrm{T}-\mathrm{H}$ characteristics to detect specific component characteristics changes. Diagnostics with (1) is referred to as plant-level diagnostics, while diagnostics with (2) or (3) is referred to as component-level diagnostics.

The "simpler" T-H formulae of set (1) can be expressed in terms of IF-THEN rules, but the more "complicated" formulae of sets (2) and (3) require the additional flexibility of the ANN knowledge structure. Table 1 shows the delineation between the ES and the ANN. In the ES (Reifman et al., 1994), the threestep process can be transformed into a three-knowledge database structure. The three databases, Physical Rules Database (PRD), Component Classification Dictionary (CCD), and PID, are independent of each other. Based on the three conservation 
equations, qualitative analysis theory yields a set of firstprinciples IF-THEN rules which are plant- and T-H systemindependent. These are grouped in the PRD. These rules utilize only the initial trends of the T-H signal variables to provide correlations at the plant level with imbalances in one of the T-H functions, $Q_{\text {mass }}, Q_{\text {mom }}$, or $Q_{\text {eng. }}$ The $C C D$ contains the classification of generic component types by the three $\mathrm{T}-\mathrm{H}$ functions. This database is, therefore, also plant- and T-H system-independent. The PID represents the component and instrumentation locations in the T-H system. This database is, therefore, the only plant- and $\mathrm{T}-\mathrm{H}$ system-dependent database in the ES. In the ANN, the hierarchy is further divided into two sublevels. The generic component characteristics formula used to classify components by the absence of generic characteristics are grouped in the network of generic component ANNs. Specific component characteristics formulae are grouped in the network of specific component ANNs. In addition to the ANN training data required, the ANN topologies may also vary from plant to plant and T-H system to T-H system. While the main division between the ES and the network of ANNs is as discussed here, there are places where IF-THEN rules can be used for compcnent-level diagnostics and places where ANNs can be used for plant-level diagnostics.

\section{PRODIAG CODE DEVELOPMENT}

While the theory underlying the PRODIAG diagnostic strategy is comprehensive and self-contained, a number of issues were encountered during the actual implementation into PRODIAG. The major issues, which primarily pertain to dynamic effects, definition of T-H loops for the ES, and data requirements for the ANN are discussed here (Reifman et al., 1995).

The transient response of a thermal-hydraulic system incorporates several dynamic effects driven by various thermalhydraulic phenomena (Reifman and Wei 1995b). These effects can be complicated to decompose or to separate and are further complicated by coupling of the phenomena. Timescales for these phenomena can also vary from the very short to the very long. Identification of a unique component malfunction from the transient response requires a classification of the various dynamic effects. One generic classification of these dynamic effects is the following: (1) Natural T-H Response, (2) Control System Response, and (3) Instrumentation Response.

This decomposition of the response into these three classes of dynamic effects is necessary if identification of a unique component malfunction or initiator of the transient is to occur, but it is not sufficient. Some methodology has to be invoked to use the dynamic features of the T-H signals to map the T-H response into these classes.

Part of that methodology involves the introduction of the concept of a Time Window Selector (TWS). The TWS opens and closes various time windows within which various definitive combinations of specific T-H signals can be correlated or rejected. The correlations depend upon the natural time constants or delays of the T-H system and the time constants of the instrumentation. Recognition of control system response through changes in signal trends is also used in the opening and closing of time windows. Details are available in (Project Staff, 1995). The other part of the methodology used to map these dynamic features of the signals to the three classes of dynamic effects requires the recognition of the occurrence of a component malfunction and the accompanying signal processing procedure (Reifman and Wei, 1995b). Currently threshold criteria are used to detect that a signal $x$, which was previously constant $\left(x^{*}\right)$, is changing $\left(x^{/-}\right)$. Other subissues regarding dynamic effects are discussed in (Reifman et al., 1995).

We now turn to the decomposition of the T-H system into loops. In accordance with the analytic decomposition strategy laid out in Table 1, the ES diagnostics utilizes only the initial trend of the temporal T-H signals but correlates them spatially across the plant to identify the component malfunction. This requires ES rules or correlations to be developed for a wide set of geometrical configurations. For these rules to be generic, the geometrical configurations have to be generic. This then necessitates the definition of a few basic configurations which have to be "simple" to enable them to be used as building blocks. All T-H systems should then te decomposable into these building blocks which will be termed as loops. To decompose a T-H system into "independent" loops requires the identification of end conditions with flows or pressures, which are "essentially" constant during a T-H transient and allows a loopby-loop decoupling to be performed during the diagnostic reasoning. A loop is defined as a continuous fluid circuit with monodirectional incompressible flow between two end conditions. This is in the direction of the monotonically decreasing pressure gradient. An open loop starts and ends at two separate end conditions. A closed loop starts and ends at the same location. Boundary condition is defined as an end condition of constant $w$ or $p$. Another example of an end condition is a tank which is a volume with separated liquid and gas phases (i.e., a level exists). The ES rules are then applied to one loop at a time. They correlate the initial trends of the signals spatially along the loop to allow an inference to be drawn. The rules and correlated reasoning in the ES for the diagnosis of the T-H response are based upon the quasi-static approximations of the three conservation equations of mass, momentum, and energy (Reifman and Wei, 1995a). Dynamic effects are treated by the TWS.

However, end conditions alone are insufficient for the T-H system decomposition. A number of other knowledge concepts are necessary to allow the decomposition of a T-H system into loops. These knowledge concepts all pertain to loop interfacing, loop connections, and loop ends. There can be hydraulic interfaces and thermal interfaces between loops. Loops can be hydraulically connected through a junction, at an ECS or at an ICS. A junction is a multiple connection of piping. Junctions are classified as components with a mass source or mass sink capability for the loop which is being diagnosed. An ECS is a $\mathrm{T}$-H system which is hydraulically connected to the specific $\mathrm{T}-\mathrm{H}$ system under diagnosis only at one location but it is valved off at normal steady state. An ICS is a T-H subsystem which is 
hydraulically part of the specific T-H system under diagnosis and is not valved off at normal steady state, but at normal steady state, the interconnecting flow is zero. Thermal interfacing of loops is treated by using the concept of an SS. A SS is a T-H system which is coupled to the specific T-H system thermally but not hydraulically at normal steady state. With these concepts, certain simple hydraulic loops can now be defined. There are the model loops into which the specific T-H system is decomposed. ES rules and the accompanying flow logic have been derived which are applicable to all these types of loops. With this ability to decompose a T-H system into these types of model loops, we are able to use a small set of generic rules and generic flow logic to apply the rules. Details are available in (Reifman et al., 1995).

We now turn to the ANN. The hierarchy of ANNs is intended to continue the diagnostics where the ES leaves off with the utilization of more complex T-H "numerical" formulas (Project Staff, 1995). We use "numerical" in quotes because in our knowledge-base structure, neural networks would be used first to perform a limited form of quantitative analysis before detailed mathematical simulation models would be used to perform detailed quantitative analysis. We become progressively more quantitative with the usage of the neural networks, but are initially looking to build a stand-alone-parts-of-a-plant model using ANNs. This use of ANNs to build a parts-of-a-plant model essentially implies that significantly more "modeling" (training) data are required over the ES. This increase in data requirements over the ES has led to a limited implementation of the ANNs, as a limited amount of "modeling" data is available. This, therefore, calls for a modular programming approach with hooks put in place for future generic and specific components introduced by different $\mathbf{T}-\mathrm{H}$ system applications.

We now turn to detailing the ANN implementation developed to this point for PRODIAG. It performs diagnostics at the plant level to supplement the ES diagnostics. The role of the plantlevel $A N N$ is to aid in the diagnosis of $Q_{\text {mass }}^{\prime-}$ or $Q_{\text {mom }}^{\prime-}$ malfunctions through the use of component characteristics. The concept of component characteristics requires definition. Component characteristics are similar to input-output relations in control theory. For our application, the functional input-output relationships are between one $\mathrm{T}-\mathrm{H}$ variable and the other $\mathrm{T}-\mathrm{H}$ variables for the component. We use here the momentum characteristics

$$
\Delta p=f(w)=\text { pressure difference across component. }
$$

We illustrate the use of the technique of component characteristics for ${Q^{\prime}}^{-}$mass and $Q^{\prime-}$ mom plant-level diagnostics with an open loop configuration. For an open loop with two end conditions and no junctions, the quasistatic momentum equation is

$$
p_{1}-p_{2}=f_{1}(w)+f_{2}(w)
$$

The loop has been divided into two segments. Segment 1 has momentum characteristic $f_{1}(w)$ while segment 2 has momentum characteristic $f_{2}(w)$. Each segment's momentum characteristic is a combination of the component characteristics in the segment. The two end conditions are at pressures $p_{1}$ and $p_{2}$, respectively, and the flow is from $p_{1}$ to $p_{2}$. The flow $w$ is measured in segment 1. Manipulating Eq. (2) gives us

$$
\left(p_{1}-p_{2}\right)-f_{1}(w)=f_{2}(w)
$$

If there is a pressure transducer which measures the pressure $p$ at the intersection of segments 1 and 2, then by Eq. (1) which is the definition of momentum characteristics, we have for segment 2

$$
\Delta p=f_{2}(w)=p-p_{2}
$$

We also have from Eqs. (3) and (4)

$$
\Delta p=\left(p_{1}-p_{2}\right)-f_{1}(w)
$$

If we plot Eq. (5) in $\Delta p$ w space, we have a curve 1 which will intersect with a plot of Eq. (4) in $\Delta p$ w space, denoted as curve 2. The intersection point is the operating point, as it gives the value of $\Delta p$ and $w$ in steady state normal operation of the open loop. A momentum malfunction, $\alpha^{-}$mom in segment 1 alters the momentum characteristic $f_{1}(w)$. This changes curve 1 in $\Delta p w$ space. However, the momentum characteristic $f_{2}(w)$ does not change, so curve 2 remains the same. The operating point, therefore, moves along curve 2. If an ANN is trained to recognize curve 2 , which is the component characteristic $f_{2}(w)$, then this ANN can be used to recognize $Q^{-}$mom malfunctions in segment 1. However, mass malfunctions $Q_{\text {mass }}^{-}$in segment 1 would also alter $f_{1}(w)$ so there would be an ambiguity in the diagnostic resolution. If the $Q^{-}$mass malfunction is in between the flow measurement and the pressure measurement, this would effectively also change $f_{2}(w)$, so the operating point would move off both curve 1 and curve 2. This type of diagnostic behavior in $\Delta \mathrm{p}$ w space allows the following conclusions to be drawn.

- No malfunctions $\rightarrow$ operating point does not move;

- $Q^{\prime}$ mass or $Q^{-}$mom in segment $2 \rightarrow$ operating point traces out curve 1;

- $\mathrm{Q}_{\text {mass }}^{\prime}$ (upstream of $\mathrm{w}$ measurement) or $\mathrm{Q}_{\text {mom }}^{-}$in segment 1 $\rightarrow$ operating point traces out curve 2 ; and

- $\mathrm{Q}^{-}$mass (downstream of $\mathrm{w}$ measurement) in segment $1 \rightarrow$ operating point traces out neither curve 1 or curve 2.

With this representation of malfunctions, an ANN can be constructed to recognize the component characteristic combinations and, therefore, support the diagnosis of $Q_{\text {mass }}^{-}$and $\alpha^{-}{ }_{\text {mom }}$ malfunctions at the plant level. This illustrates the PRODIAG ANN plant-level diagnostic technique for a model open loop configuration. This technique has been implemented for a subsystem of the Braidwood CVCS configuration.

Figure 1 shows the CVCS test system used for the proof-ofconcept. Focusing on the subsystem downstream of the volume 
control tank (VCT) shows that there is a central seal water closed loop with three branches off the loop. The closed loop consists of junction 5 downstream off the VCT; passes through charging pump $A$; then into the reactor coolant system (RCS) pump represented within the dashed enclosure by the seal barriers, the radial bearing and seal \#1; and back through the seal water heat exchanger (HX) to junction 5 . Branch 1 is formed by the loop from the VCT to junction 5 ; branch 2 is formed by the charging loop from junction 7 , through the regenerative $\mathrm{HX}$ back to the RCS cold leg; while branch 3 is formed by the RCS seal barrier leakage loop from junction 8 to the RCS cold leg. Flow meter FT 121 measures $w_{I}$ flow, while flow meter FE-145 measures $w_{\text {II }}$ flow. Application of the method of component characteristics, illustrated above for the open loop configuration, to the CVCS configuration leads to the following ANN representation in $\mathrm{w}_{\mathrm{I}} \mathrm{w}_{\mathrm{II}}$ space.

- $Q_{\text {mom }}^{\prime}$ malfunctions in segment $\mathbf{1} \rightarrow$ operating point traces curve 1;

- $\mathrm{Q}_{\text {mass }}^{\prime-}$ or $\mathrm{Q}_{\text {mom }}^{-}$malfunctions in branch $2 \rightarrow$ operating point traces curve 2; and

- $\alpha_{\text {mom }}^{-}$malfunctions in segment $3 \rightarrow$ operating point traces curve 3.

Segment 1 is that part of the closed loop between junction 5 and junction 7, while segment 3 lies between junction 7 and junction 8. Curves 1-3 are combinations of the momentum characteristics for different segments and branches. ANNs have been constructed to recognize these curves given the "model" data for the Braidwood CVCS. This is discussed in the next section. It can be seen that a significant amount of "modeling" data is required for the various momentum characteristics.

\section{DIAGNOSTICS TESTING RESULTS}

Initial results obtained by using the initial "first-principles ES" portion of the PRODIAG code to diagnose transient events were summarized in (Reifman et al., 1994). Additional ES validation results have been obtained and are reported here. The validation matrix consists of both semi-blind and blind testing. In the case of the semi-blind testing, the identification of the simulator testing transients were known a'priori, but this knowledge was not used during the testing. For the blind testing the a'priori identification of the simulator testing transients was not made available. Some testing of the ANN representations was also performed. We repeat here the description of the specific $T-H$ test system from (Reifman et al., 1994). The Braidwood nuclear power plant is a twin-unit PWR (Pressurized Water Reactor) with each PWR rated at $1175 \mathrm{MWe}$. The Braidwood CVCS was selected as the test bed for the development of the process diagnostics code PRODIAG.

One particular CVCS configuration for one specific operating mode was selected. This configuration is normal charging/letdown mode at $100 \%$ power. Simplifications can therefore be made in the CVCS system layout. Figure 1 shows the simplified CVCS PID used for this project. Except for
Makeup and Component Cooling Water all other external systems are dropped as they do not respond during the transients involved with normal charging/letdown. All external relief tanks are retained. Figure 1 also shows the location of the system instrumentation and, therefore, the plant parameter signals which can be used for the ES/ANN reasoning. Only T-H signals, excluding the external systems measurements, are utilized. The CVCS has several local controllers. It should be adequate to treat the smaller set of local controllers shown in Fig. 1. These are the TCV130A controller, PCV131 controller, VCT makeup controller, VCT hydrogen controller, and the CV121 controller. This should be the important set. All other controllers are in the manual mode.

The full-scope Braidwood simulator malfunctions for CVCS operator training are used as the initial basis for the selection of the test matrix of transients. The candidate matrix of 20 transients is shown in Table 2. Each single-fault transient event was simulated for $40 \mathrm{~s}$, including $3 \mathrm{~s}$ of null transient, starting from a steady-state normal mode of the CVCS operation with the plant at $100 \%$ nominal power.

\section{TABLE 2. OPERATOR TRAINING CANDIDATE TRANSIENT}

Charging Pump A Trip

VCT Diver Valve Failure (LCV-112A)

PCV 131 Auto Controller Failure

Clogged RCS Filter

Clogged Seal Injection Filter

Failure of Pressure Transmitter -131

Failure of Temperarure Element -130

Flow Control Valve 121 Failure

Letdown Relief Valve Fails Open

Charging Line Leak Outside Containment

Regenerative Heat Exchanger Tube Leak

Volume Control Tank Level Malfunction (LT-112)

Volume Control Tank Press Malfunction (PT-115)

Charging Header (HCV-182) Control Failure

Letdown Line Leak Outside Containment

Letdown Heat Exchanger Tube Leak

Letdown Line Leak Inside Containment

Charging Line Leak Inside Containment

Seal Injection Line Leak

Reactor Coolant Pump Seal \#1 Failure

A total of ninety-seven transients corresponding to twenty distinct single-component malfunctions have been simulated and used for testing. Thirty-nine out of the 97 transients were used to blind test the diagnostic system where the identity of the transient was not provided until after the analysis. Table 3 shows that, out of the 39 transients 37 were correctly identified within the first 40 seconds into the transient with graded degree of accuracy, i.e., uniquely identified, identified as one of two candidates, etc. The remaining two transients, with very mild severity levels, were not identified. Nineteen transients, corresponding to $49 \%$ of the simulated transients are uniquely 


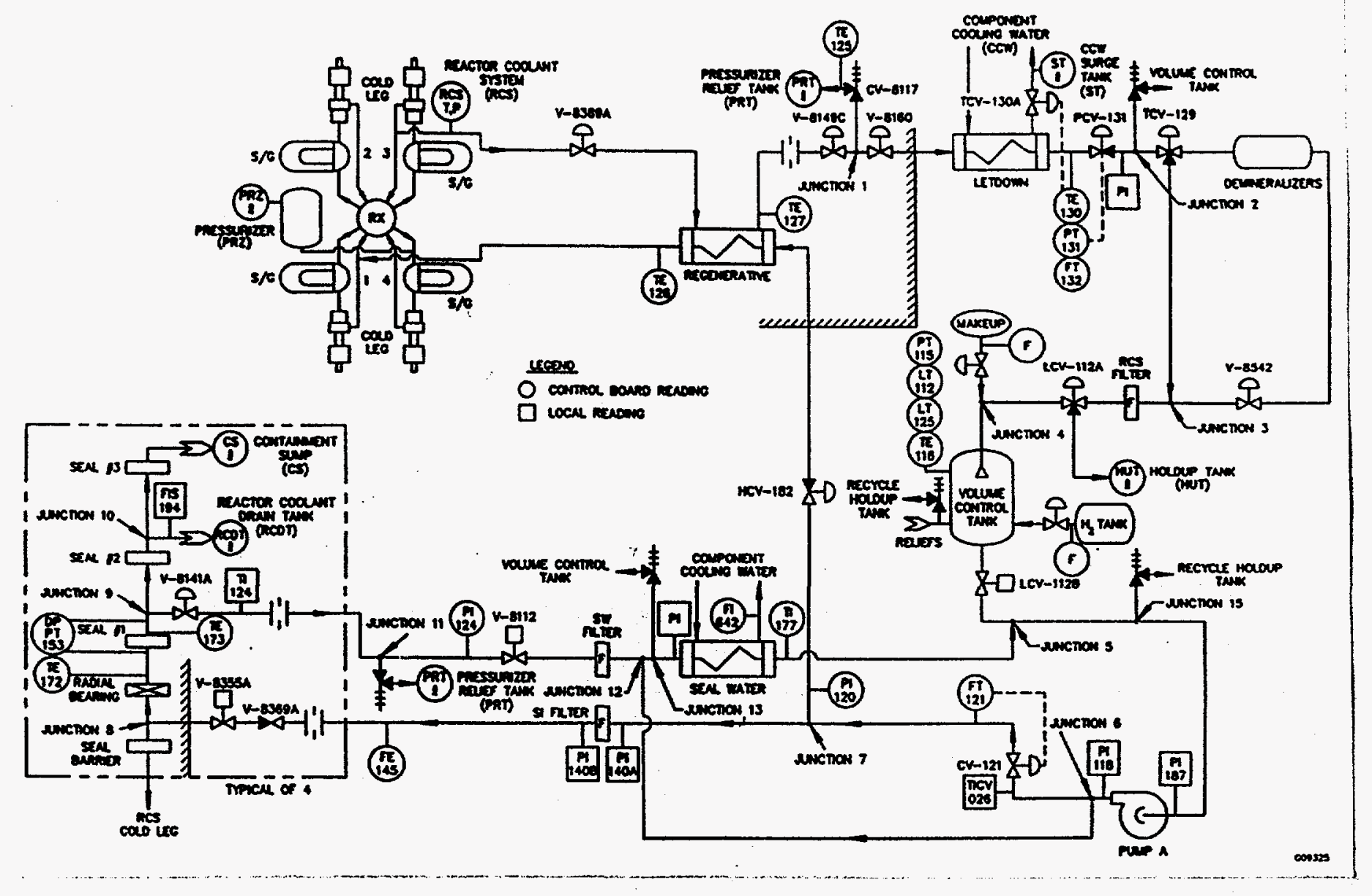

FIG 1. PROOF-OF-CONCEPT CVCS TEST SYSTEM

TABLE 3. SUMMARY OF PRODIAG BLIND TEST PERFORMANCE

\begin{tabular}{c|c|c|c|c|c}
\hline $\begin{array}{c}\text { Uniquely } \\
\text { Identified }\end{array}$ & $\begin{array}{c}2 \text { Possible } \\
\text { Candidates }\end{array}$ & $\begin{array}{c}3 \text { Possible } \\
\text { Candidates }\end{array}$ & $\begin{array}{c}4 \text { or more } \\
\text { Candidates }\end{array}$ & $\begin{array}{c}\text { Incorrect } \\
\text { Diagnostics }\end{array}$ & $\begin{array}{c}\text { No } \\
\text { Diagnostics }\end{array}$ \\
\hline $\begin{array}{c}19 \\
(49 \%)\end{array}$ & $\begin{array}{c}3 \\
(8 \%)\end{array}$ & $\begin{array}{c}13 \\
(33 \%)\end{array}$ & - & 2 \\
$(5 \%)$ & $(5 \%)$ \\
\hline
\end{tabular}

identified, three transients or $8 \%$ are identified as one out of two possibilities, 13 transients or $33 \%$ are identified as one out of three, two transients or $5 \%$ are identified as one out of four possibilities, and two or $5 \%$ are not identified. As discussed in the section above, instrumentation failure should be detected by the classical techniques of signal validation. The two unidentified simulations refer to the pressure transmitter failure of instrument PT-CV131 which cannot be detected. Overall, PRODIAG correctly diagnosed $95 \%$ of the transients with varying precision and did not identify $5 \%$ of the transients. No transients were misclassified. These results indicate that the PRODIAG system is a significant step in the direction of addressing the major limitations of existing AI-based advisory systems.

Testing of the component characteristics approach using ANNs developed for PRODIAG is shown in Fig. 2. These results were obtained for the CVCS configuration discussed in the previous section, which addressed the plant-level diagnostics question of whether the malfunction is $Q_{\text {mass }}{ }^{1-}$ or $Q_{\text {mom }}{ }^{1-}$. The method of component characteristics is used to give curves 1-3 in $w_{1} w_{U}$ space with each curve corresponding to a certain set of malfunctions.

The $w_{1}$ flow is measured at instrument location FT-121 and the flow $w_{I I}$ is measured at location FE-145. Figure 2 shows the 


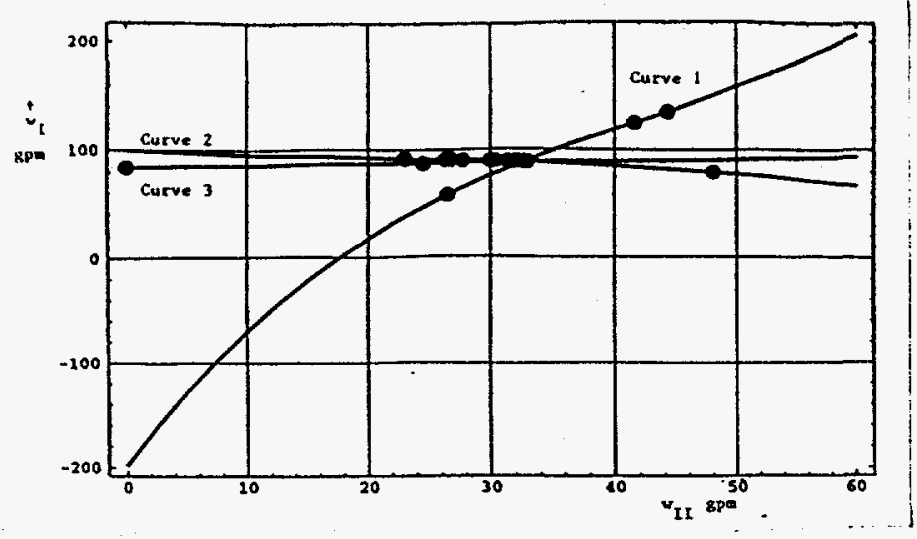

FIG 2. TEST OF ANN REPRESENTATIONS FOR CVCS SEAL LOOP MALFUNCTIONS

actual data from the set of testing transients as dots. It can be seen that the group of dots do actually congregate around the set of three curves in $w_{I} w_{I I}$ space. The fact that the transient data does group around the predicted three curves shows that the method of component characteristics is a valid one. ANNs trained to associate particular $\mathrm{Q}_{\text {mass }}$ or $\mathrm{Q}_{\text {mom }}$ failures in segment 1 , branch 2 and segment 3, with curves 1-3 were tested for various transients. In every case, the correct diagnosis was provided.

\section{CONCLUSIONS}

The hybrid knowledge structure based on the combined use of a first-principles ES and component-characteristic ANNs has been constructed for the diagnostic system. Implementation in the PRODIAG code has been completed for a laboratory proofof-concept system. From the validation results of the laboratory testing at the CRADA phase of "proof-of-concept," the proposed approach appears promising. A set of first-principles physics based ES rules and a supervisory flow logic which are plant and T-H system independent have been developed. While ANN training has to be T-H system specific, a set of ANN representations which could be derived by a plant- and T-H system-independent automated reasoning program, has also been developed. The objective of generic portability appears to be feasible. Further testing will be performed with another T-H system from a PWR, such as the Component Cooling Water System. Signal processing issues involving plant data will have to be assessed. The issue of optimization for time performance has been addressed to some extent. Future work will also extend the range of applicability.

Although the proposed computer-based diagnostic system will be applied to the diagnosis of component failures in nuclear power plants, the approach used is general and has a wide range of applications. In addition to the electric power industry (both nuclear and fossil), the proposed general-purpose approach for process diagnosis can be applied to numerous processes in the chemical industry. If the proposed concepts of this advanced diagnostic system can be scaled up, the application of the proposed technology could be of utility in various industries.

\section{ACKNOWLEDGMENTS}

We wish to thank Mark Olsen of the Commonwealth Edison Braidwood Station for providing the full scope operator training simulator results. Acknowledgment is due to Karen Jenicek for her careful typing of this paper.

Work supported by the U.S. Department of Energy, Energy Research-Laboratory Technology Transfer Program, under contract W-31-109-ENG-38.

\section{REFERENCES}

Bernard, J. A., and Washio, T., 1989, Expert Systems Applications Within the Nuclear Industry, American Nuclear Society, Chicago.

Kirk, D. B., and Murray, A. E., 1988, "Verification and Validation of Expert System for Nuclear Power Plant Applications," Technical Report EPRI-NP-5978, Electric Power Research Institute, Palo Alto, California.

Reifman, J., Briggs, L. L., and Wei, T. Y. C., 1991a, "A First-Principles General Methodology for Representing the Knowledge Base of a Process Diagnostic Expert System," Proceedings, 4th International Conference on Industrial and Engineering Applications of Artificial Intelligence and Expert System, Kauai, Hawaii, pp. 255-265.

Reifman, J., Briggs, L. L., and Wei, T. Y. C., 1991b, "Nuclear Power Plant Diagnostics Using Qualitative Analysis and Component Functional Classification," Proceedings, A191: Frontiers in Innovative Computing for the Nuclear Industry, Jackson, Wyoming, pp. 227-236.

Reifman, J., Wei, T. Y. C., Abboud, R. G., and Chasensky, T. M., 1994, "Cooperative Research and Development for Artificial Intelligence Based Reactor Diagnostic System," Proceedings, The American Power Conference, Chicago, II, Vol. 56-I, pp. 365-370.

Reifman, J., Wei, T. Y. C., and Vitela, J. E., 1995, Argonne National Laboratory, unpublished information, Argonne, IL.

Reifman, J., and Wei, T. Y. C., 1995a, "Systematic Construction of Qualitative Physics-Based Rules for Process Diagnostics," Proceedings, 7th Portuguese Conference on Artificial Intelligence, Funchal, Madeira Island, Portugal, pp. 311-322.

Reifman, J., and Wei, T. Y. C., 1995b, "PRODIAG Dynamic Qualitative Analysis for Process Fault Diagnosis," Proceedings, 9th Power Plant Dynamics, Control and Testing Symposium, Knoxville, Tennessee, pp. 40.01-40.15. 